\title{
Hedge fund portfolio selection with modified expected shortfall
}

\author{
K. Boudt ${ }^{1}$, B. G. Peterson ${ }^{2} \&$ P. Carl $^{3}$ \\ ${ }^{1}$ FWO, Flanders and K.U.Leuven, Belgium \\ ${ }^{2}$ Diamond Management \& Technology Consultants, Chicago, IL, USA \\ ${ }^{3}$ Guidance Capital Management, Chicago, IL, USA
}

\begin{abstract}
Modified Value-at-Risk (VaR) and Expected Shortfall (ES) are recently introduced downside risk estimators based on the Cornish-Fisher expansion for assets such as hedge funds whose returns are non-normally distributed. Modified VaR has been widely implemented as a portfolio selection criterion. We are the first to investigate hedge fund portfolio selection using modified ES as optimality criterion. We show that for the EDHEC hedge fund style indices, the optimal portfolios based on modified ES outperform out-of-sample the EDHEC Fund of Funds index and have better risk characteristics than the equal-weighted and Fund of Funds portfolios. Keywords: portfolio optimization, modified expected shortfall, non-normal returns.
\end{abstract}

\section{Introduction}

It is generally accepted that rational investors should allocate their portfolio optimally according to a return/risk criterion. The most widely studied criteria (minimum variance and maximum Sharpe ratio) are based on the first two portfolio moments. A more general set of positive preferences for odd moments (mean, skewness) and aversion to even moments (variance, kurtosis) is discussed by Scott and Horvath [1]. For largely intuitive reasons, the relationships between the moments themselves are often summarized into risk measures. In the presence of non-normal returns and when investors have a non-quadratic utility function, two issues arise: (i) the choice of risk measure and (ii) the estimator for this risk measure. 
The literature on portfolio risk measurement seems to agree that, in the presence of non-normal returns and non-quadratic utility functions, a downside risk measure should be used rather than the portfolio variance which gives an equal weight to positive and negative returns. Value-at-Risk (VaR) and Expected Shortfall (ES) are the most popular downside risk predictors. VaR is the negative value of the portfolio return such that lower returns will only occur with at most a preset probability level denoted $\alpha$, which typically is between 1 and 5 per cent. Expected Shortfall is the negative value of the mean of all return realizations that are below the VaR. More formally, let $F(\cdot)$ and $F^{-1}(\cdot)$ be the distribution and quantile function of the portfolio return $r_{p}$ and assume that they are continuous. Then VaR and ES with loss probability $\alpha$ are defined as follows:

$$
\begin{aligned}
\operatorname{VaR}(\alpha) & =-F^{-1}(\alpha) \\
\operatorname{ES}(\alpha) & =-E_{F}\left[r_{p} \mid r_{p} \leq F^{-1}(\alpha)\right] .
\end{aligned}
$$

When portfolio returns are asymmetric, VaR may no longer be an acceptable risk measure in a portfolio context because it fails to be "subadditive". When VaR is used the risk of the overall portfolio could be higher than the combined risks of the single positions in spite of portfolio diversification effects. Moreover, because VaR is not a convex function of the portfolio weights, mean-VaR portfolio optimization is a difficult programming task. Expected Shortfall, on the contrary, is a coherent risk measure and is a convex function of the portfolio weights (Bertsimas et al. [2]). Its widespread implementation on portfolios of non-normal returns has been hampered by the fact that before our paper [3], there was no fast-to-compute estimator for ES that performs well when returns are skewed and heavy tailed.

Regarding the issue of the estimation of portfolio VaR and ES, note first of all in eqns. (1) and (2) that these risk measures are defined as a function of the portfolio return distribution function and that this distribution function is unknown for real time series. Very often, VaR and ES are computed under the hypothetical assumption of normality. The resulting estimators are called Gaussian VaR and ES. It is a stylized fact that the returns on many financial assets are not normally distributed. It follows that methods that take into account the non-normality of the true return distribution will produce more accurate downside risk estimates than Gaussian VaR and ES do. One such very popular methodology relies on using the Cornish-Fisher and Edgeworth expansions to estimate the distribution function as the normal distribution plus some correction terms that account for the skewness and excess kurtosis observed in the data. The resulting estimators are called modified VaR and modified ES. Today modified VaR is, among practitioners, one of the most popular estimators for evaluating the risk of hedge funds and other non-normally distributed assets and has been widely used as a portfolio selection criterion (see e.g. Favre and Galeano [4]). Motivated by the desirable mathematical properties of ES and the widespread interest in modified VaR, we have shown in a recent paper [3] how the Edgeworth and Cornish-Fisher expansions of the density and quantile functions can be used to obtain an estimator for ES, called modified ES, that delivers accurate downside risk estimates even in the presence 
of non-normal returns. This paper studies the use of modified ES as an objective for the construction of a fund of hedge funds based on the EDHEC hedge fund style indices.

The remainder of the paper is organized as follows. In Section 2 we describe our portfolio optimization strategy, introduce the data and present our methodology for solving the portfolio selection problem. Results are discussed in Section 3. Finally, Section 4 concludes and outlines the implications for further research.

\section{Data and methodology}

\subsection{Data}

We apply our portfolio construction techniques to the January 1997-December 2007 monthly return series of the EDHEC hedge fund investment style indices (132 observations). EDHEC style indices are constructed such that the returns on these indices reflect the monthly performance of hedge funds pursuing that particular style. (The EDHEC indices are constructed using PCA factor replication against other published hedge hedge fund indices (HFR, CSFB, etc.) and private databases of hedge fund returns. In this manner, they attempt to correct for the biases of the individual hedge fund index construction methodologies and construct a "meta-index" that is more broadly representative of the actual performance of a particular style.) The monthly return on the EDHEC Fund of Funds index is symbolically representative of the performance of the Fund of Hedge Funds providers. The comparison to the Fund of Funds index here is meant to be illustrative; these are general observations about performance of optimized portfolios and the comparison against the Fund of Funds index is merely intended to provide context.

Table 1 reports the main descriptive statistics for each of these series, which will be of importance for the optimal portfolio allocations. From an investor's perspective, the equity market neutral style index has the best combination of statistical properties: a relatively high Sharpe-ratio, low volatility, positive skewness and a low downside risk as measured by the $95 \%$ modified VaR and ES statistics. Clearly, assets with negative skewness should have a higher downside risk than assets with positive skewness and the higher the excess kurtosis, the higher downside risk is for negatively skewed return distributions. In Table 1 we see that modified VaR and ES respect these intuitive conditions for being a reliable downside risk estimate for non-normal returns. Indeed, note that Merger Arbitrage and Relative Value have a similar mean return and standard deviation, but Merger Arbitrage has much more negative skewness and higher kurtosis than Relative Value. Consequently, Merger Arbitrage's modified VaR and ES are higher than Relative Value's modified VaR and ES. Note however that the overall level of modified VaR and ES is determined mostly by the asset's mean and standard deviation, while skewness and excess kurtosis have only a second order impact on modified VaR and ES unless either have extreme values. 
Table 1: Descriptive statistics for the 1997-2007 monthly return series of the EDHEC hedge fund style indices.

\begin{tabular}{lrrrrrr}
\hline & Mean & Std.Dev. & Skew. & Exc.Kurt. & $\mathrm{mVaR}(0.05)$ & $\mathrm{mES}(0.05)$ \\
\hline Convertible Arbitrage & 0.007 & 0.011 & -0.835 & 1.204 & 0.014 & 0.022 \\
CTA Global & 0.006 & 0.026 & 0.086 & -0.208 & 0.035 & 0.044 \\
Distressed Securities & 0.010 & 0.015 & -1.732 & 9.778 & 0.018 & 0.059 \\
Emerging Markets & 0.011 & 0.036 & -1.378 & 7.098 & 0.055 & 0.139 \\
Equity Market Neutral & 0.007 & 0.006 & 0.279 & 0.815 & 0.002 & 0.005 \\
Event Driven & 0.009 & 0.016 & -1.872 & 9.435 & 0.021 & 0.062 \\
Fixed Income Arbitrage & 0.005 & 0.010 & -5.036 & 37.567 & 0.013 & 0.013 \\
Global Macro & 0.009 & 0.017 & 0.922 & 1.830 & 0.014 & 0.018 \\
Long Short Equity & 0.009 & 0.020 & -0.005 & 0.899 & 0.023 & 0.034 \\
Merger Arbitrage & 0.007 & 0.011 & -1.749 & 7.252 & 0.013 & 0.035 \\
Relative Value & 0.008 & 0.010 & -1.046 & 2.586 & 0.010 & 0.020 \\
Fund of Funds & 0.008 & 0.016 & 0.161 & 3.226 & 0.017 & 0.027 \\
\hline
\end{tabular}

\subsection{Portfolio optimization problem}

We design our portfolio optimization problem to be a stylized representation of actual practice in the fund of hedge funds world. In our exploratory model, investors are either pure risk minimizers or reward/risk maximizers. They use either the portfolio standard deviation or modified Expected Shortfall as risk measures and take the generalized Sharpe ratio defined as the mean return divided by the risk measure as a reward/risk criterion. We rebalance these portfolios annually, as this is symbolically consistent with the actual longer holding periods and lock-ups common in Funds of Funds. Finally, we impose minimum $2.5 \%$ and maximum $35 \%$ bound constraints on the portfolio weights to avoid "edge" portfolios. We further consider the equal-weighted portfolio and the EDHEC Fund of Funds index as benchmark portfolios to evaluate the performance of our minimum risk and maximum reward/risk portfolios against.

Hedge fund portfolio selection under these criteria requires the solution to a linearly constrained nonlinear optimization problem. Because many local optima points exist to this problem, we have undertaken the two-step approach of (i) bruteforce exploration of the feasible space using a step size of 0.05 , where we compute for each possible combination of portfolio weights the value of the objective function and save the ten best solutions; followed by (ii) local optimization of the objective function using the DONLP2 sequential quadratic programming algorithm initialized at the ten best solutions obtained via the grid search of step (i). The solution to the optimization problem is the global optimum of these ten local optima. This is clearly a very greedy optimization method, since it consists in evaluating the optimization criterion for hundreds of thousands of potential 
portfolios. In Boudt et al. [3], we showed that all the optimization criteria can be expressed as analytical and fast-to-compute formulae of multivariate moments, which eases the optimization task somewhat. (The data, functions and figures used in this paper are available in the R package PerformanceAnalytics [5] or directly from the authors.)

The risk and reward/risk criteria need to be estimated. We account for the potential time-variation in the distribution of the hedge fund return series by estimating these criteria on the basis of a rolling three year estimation sample. Since the history of the data set starts on January 1997, we thus dispose of out-ofsample returns of the optimally designed portfolios for the period January 2000 December 2007.

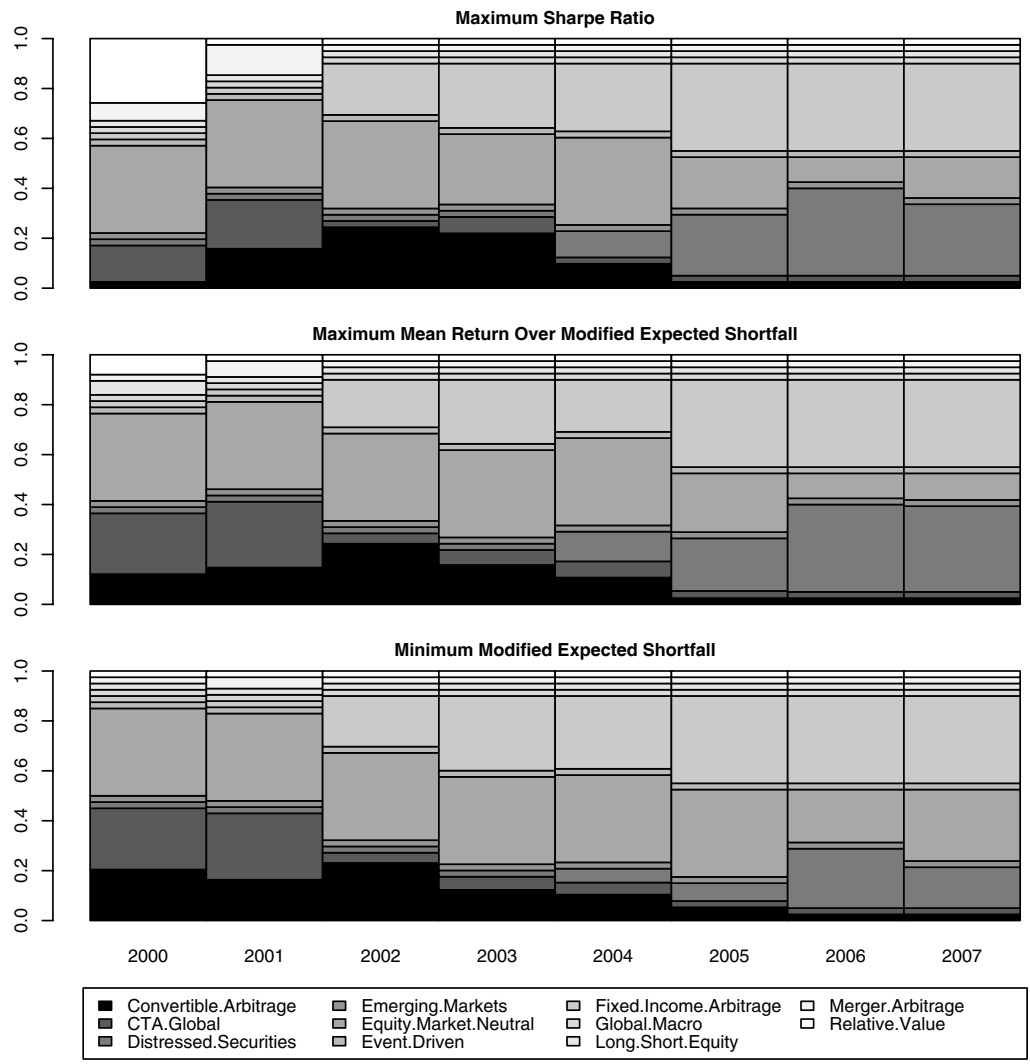

Figure 1: Weight allocations for yearly rebalanced portfolios of EDHEC indices. 


\section{Results}

\subsection{Portfolio allocation}

The stacked bar weights charts in Figure 1 show that the maximum Sharpe ratio, minimum modified ES, and maximum mean return over modified ES optimization criteria all produce very similar portfolios for the years 2000-2007. Allocations tend to go to low-standard deviation strategies, such as Equity Market Neutral, Convertible Arbitrage and Fixed Income Arbitrage. All three objective functions allocate to CTA global and Convertible Arbitrage at the beginning and then shift into Distressed Securities starting in 2004.

The strategies that receive only a minimum allocation include Emerging Markets, Event Driven, Long-Short Equity, Merger Arbitrage, and Relative Value. For the most part, the omission of these strategies can be explained by their higher standard deviation and (sometimes strongly) negative skewness and positive kurtosis. Where standard deviation may not seem particularly high, such as Long Short Equity, the strategies can be characterized as having lower return to risk ratios, in addition to negative skewness and positive kurtosis.

Fixed Income Arbitrage does not receive much of an allocation in the first two years, and a review of the rolling three-year standard deviation, skewness and kurtosis makes it clear why. The surge in these statistics for this strategy during the Russian crisis and LTCM carry through the analysis until 2001, when that period drops out of the rolling window. In actual practice it may be desirable to use a regime switching model or conditional risk model to condition the moments for use in analysis.

Equity Market Neutral tends to be a cornerstone of portfolios generated like these because of the strategy's relatively high ratio of reward to standard deviation and sometimes positively skewed distributions. The mean return and (positive) skewness to the strategy index seem to drop considerably in 2003, which results in lower allocations in the max SR and min modified ES portfolios. The allocation shrinks somewhat less in the mean return over modified ES portfolio, where it is preferred over Distressed Securities.

CTA Global receives a considerable weight for the years 2000-2001. As a strategy, however, CTA Global is characterized as a lower-Sharpe ratio strategy given its higher standard deviation. For these years, however, the standard deviation is somewhat lower and the correlation of the CTA Global index is near zero or negative (to -0.5 ) to some indices. The small or even negative correlation is probably the factor explaining the presence of CTA in the portfolios for the first two or three years. Once the rolling correlation level rises, the strategy receives only the minimum allocation.

The most notable difference in the portfolios is a relatively small one. The maximum Sharpe ratio portfolio selects Relative Value in 2000 and Merger Arbitrage in 2001. The objective functions that consider higher moments tend to select near-minimum weights in those strategies during those periods, given their strongly negative skewness and kurtosis measures coming from the crises of 

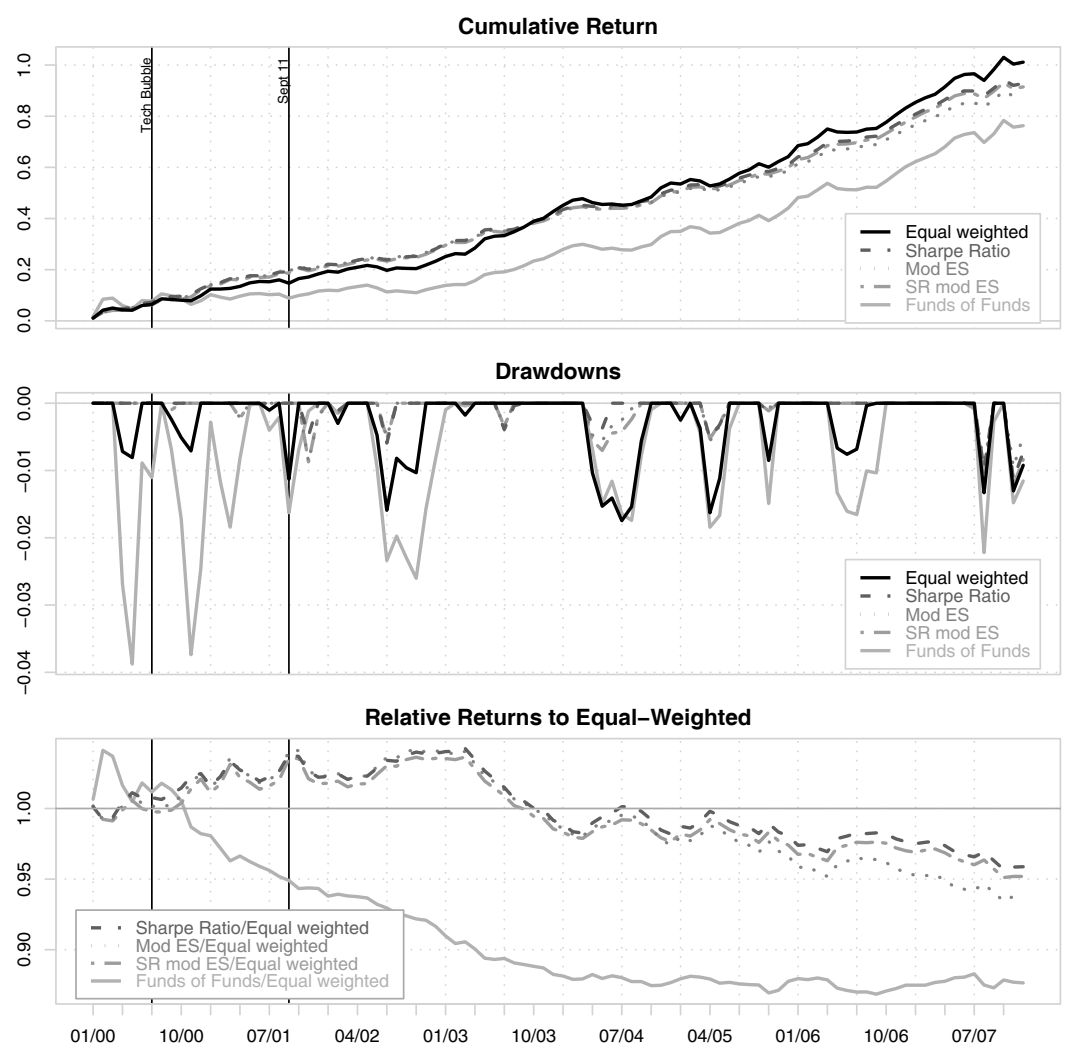

Figure 2: Out-of-sample cumulative portfolio return charts.

1998. Even after the measures of those higher moments fall, however, none of the objective functions allocate to those strategies.

\subsection{Portfolio performance}

The first panel of Figure 2 shows the cumulative performance of the different portfolios and the EDHEC Fund of Funds index. The portfolios tend to outperform the Fund of Funds index, although the portfolio performance is not shown net of the additional fees that funds of hedge funds charge. Were those fees added, we might see similar performance. It is worth pointing out that the similar allocations we observed above result in very similar performance of the optimized portfolios.

The second panel of Figure 2 shows the portfolios' drawdowns, measured as a percentage of the maximum cumulative return achieved. In this case, we can see that the Fund of Funds index shows drawdowns in 2000 and 2001. Those are 
modest relative to the equity markets, of course, but are worse than any of the other portfolios. The equal weighted portfolio tends to have the next highest drawdowns. The optimized portfolios tend to have smaller drawdowns than the equal weighted portfolio. The objective functions that consider higher moments do avoid assets with negative skewness that have a higher downside risk, preferring assets with positive skewness. The advantage of considering those higher moments is somewhat unclear here, since the maximum Sharpe ratio (Markowitz) portfolio has also avoided drawdowns by selecting lower standard-deviation strategies. In the presence of non-normal returns, a maximum Sharpe Ratio strategy may not always work, depending on the co-skewness and co-kurtosis of the strategies (which may lead to higher combined portfolio variance) and the nature of the drawdown.

The third panel of Figure 2 shows the ratio of the cumulative performance for the monthly returns of the optimized portfolios and the EDHEC Fund of Funds index versus the equal-weighted portfolio. The value of the chart is less important than the slope of the line. If the slope is positive, the optimized portfolio (numerator) is outperforming the equal-weighted one, and vice versa. Note that the relative performance of the optimized portfolios versus the equal-weighted portfolio is very different in the period January 2000-December 2003 from the period January 2004-December 2007. In the first period, the optimized portfolios clearly outperform the equal-weighted portfolio, while in the second period the equal weighted portfolio outperforms the optimized portfolios. Also in the first period, the equal weighted portfolio outperforms the Fund of Funds index. In the second period, performance of the equal-weighted portfolio and Fund of Funds index is very similar (the relative performance line being flat).

The nature of the under- or out-performance to the equal weighted portfolio is instructive. The strategies that are rarely or never selected by the optimizer have been more volatile but have periods of higher performance. Those strategies more frequently selected have lower mean returns but also much lower standard deviation and/or higher (more positive) skewness. During the period following 2003, the rarely selected strategies saw rising mean returns again after their decline in the early part of the decade. Strategies selected saw mean returns remain steady or decline after 2003, with the exception of Distressed Securities.

The "hinge" in relative performance we observe in 2003 might suggest that the broader market conditions that followed provided a positive environment for those lower-Sharpe ratio strategies. The equal-weight portfolio, as it is indifferent to the issues of selection, captures the shift of relative performance. We might speculate that the attributes of high liquidity, low market volatility, and available credit for leverage all combined to help those strategies prevail during that period. Unfortunately for the performance of the optimized portfolios, this information remains outside of their preview.

\section{Conclusion}

This paper proposed and examined the use of modified Expected Shortfall as a criterion for optimal selection of portfolios of hedge funds and illustrates this 
approach for the EDHEC hedge fund style indices. Further work will examine portfolio selection in a risk budgeting framework where an explicit maximum risk contribution is specified for each portfolio component, or where the component contributions are minimized as much as possible. Additional works should examine whether different utility functions are called for in a fund of hedge funds context when optimizing individual style portfolios versus optimizing weightings across styles.

\section{References}

[1] Scott, R.C. \& Horvath, P.A., On the direction of preference for moments of higher order than the variance. Journal of Finance, 35(4), pp. 915-919, 1980.

[2] Bertsimas, D., Lauprete, G.J. \& Samarov, A., Shortfall as a risk measure: Properties, optimization and applications. Journal of Economic Dynamics and Control, 28(7), pp. 1353-1381, 2004.

[3] Boudt, K., Peterson, B.G. \& Croux, C., Estimation and decomposition of downside risk for portfolios with non-normal returns. Working paper, 2007.

[4] Favre, L. \& Galeano, J.A., Mean-modified value-at-risk optimization with hedge funds. Journal of Alternative Investment, 5(2), pp. 2-21, 2002.

[5] Carl, P. \& Peterson, B.G., PerformanceAnalytics: Econometric tools for performance and risk analysis, 2008. R package version 0.9.7. 